Rev. Int. Contam. Ambie. 35 (4) 1039-1049, 2019

DOI: 10.20937/RICA.2019.35.04.21

\title{
EFECTO DEL PRETRATAMIENTO HIDROTÉRMICO (ALTA PRESIÓN) E HIDRÓLISIS ENZIMÁTICA DE CLADODIOS DE Opuntia ficus-indica SOBRE LA LIBERACIÓN DE AZÚCARES Y SU USO POTENCIAL EN LA PRODUCCIÓN DE BIOETANOL
}

Effect of hydrothermal pretreating (high pressure) and enzymatic hydrolysis of cladodes of Opuntia ficus-indica on the release of sugars and their potential use in bioethanol production

\author{
Andrés TEXCO-LÓPEZ ${ }^{1}$, Jorge ÁLVAREZ-CERVANTES ${ }^{1}$, Arturo CADENA-RAMÍREZ ${ }^{2}$, \\ Javier CASTRO-ROSAS ${ }^{3}$, Carlos Alberto GÓMEZ-ALDAPA ${ }^{3}$ y Alejandro TÉLLEZ-JURADO ${ }^{1 *}$
}

${ }^{1}$ Departamento de Biotecnología, Universidad Politécnica de Pachuca, km 20 carretera Pachuca-Ciudad Sahagún, Ex Hacienda de Santa Bárbara, 43830 Zempoala, Hidalgo, México

2 Departamento de Ingeniería Biomédica, Universidad Politécnica de Pachuca, km 20 carretera Pachuca-Ciudad Sahagún, Ex Hacienda de Santa Bárbara, 43830 Zempoala, Hidalgo, México

3 Área Académica de Química, ICBI, Universidad Autónoma del Estado de Hidalgo, km 4.5 carretera PachucaTulancingo, 42184 Mineral de la Reforma, Hidalgo, México

*Autor para correspondencia: alito@upp.edu.mx

(Recibido: abril 2017; aceptado: junio 2019)

Palabras clave: azúcares reductores, fermentación alcohólica, hidrólisis a alta presión, hidrólisis biológica

\section{RESUMEN}

La producción de biocombustibles de segunda generación requiere de materias primas que reúnan ciertas características, entre las que destacan disponibilidad, precio, rápido crecimiento, fácil manipulación, entre otras. Opuntia ficus-indica fue analizada en este trabajo dada su amplia distribución en México, tolerancia a ambientes extremos de sequía y temperatura, así como su alto rendimiento como cultivo; aunado a esto, en periodos de poda, los cladodios son abandonados en el sitio generando problemas de contaminación por lo que es necesario generar propuestas de aprovechamiento. Se utilizaron cladodios de nopal como materia prima para la producción de etanol, utilizando un pretratamiento hidrótérmico $(\mathrm{PTH})$ en un reactor de alta presión (RAP) para favorecer la hidrólisis de mucílago y fibras. Se estudió el efecto de la temperatura (150, 175,185 y $\left.205^{\circ} \mathrm{C}\right)$, la adición de $\mathrm{H}_{2} \mathrm{SO}_{4}(0,0.5,1,1.5$ y $2 \%)$ y el tiempo de residencia en el RAP (1, 5 y 15 min). El residuo sólido obtenido del PTH fue sometido a un tratamiento enzimático, a fin de mejorar la hidrólisis y aprovechar en su totalidad esta materia prima. Finalmente se realizó una fermentación alcohólica con Saccharomyces cerevisiae. En los resultados obtenidos destaca el ahorro de tiempo por la aplicación del PTH en comparación con el pretratamiento tradicional, alcanzándose una hidrólisis final del $70.4 \%$ de los cladodios de nopal (combinación de los pretratamiento térmico e hidrólisis enzimática) y una producción de bioetanol de $6.6 \mathrm{~g} / \mathrm{L}$.

Key words: alcoholic fermentation, biological hydrolysis, high pressure hydrolysis, reducing sugars 


\begin{abstract}
The production of second-generation biofuels requires raw materials that meet certain characteristics, among which stand out availability, price, fast growth, easy manipulation, among others. Opuntia ficus-indica was analyzed in this work given its wide world distribution, tolerance to extreme environments of drought and temperature, as well as its high yield as a crop. In addition, in times of pruning, the cladodes are abandoned on the site generating pollution problems so it is necessary to generate usage proposals. The production of bioethanol from cactus cladodes has been previously proposed but a hydrothermal pretreatment (HTP) in a high-pressure reactor (HPR) has not been applied. The use of high pressures is a method used with relative success in the pretreatment of other lignocellulosic substrates. In this work, the effect of temperature (150, 175,185 and $\left.205^{\circ} \mathrm{C}\right)$, the addition of $\mathrm{H}_{2} \mathrm{SO}_{4}(0,0.5,1,1.5$ and $2 \%)$ and the residence time in a HPR (1, 5 and $15 \mathrm{~min}$ ) was studied. The solid residue obtained in the HTP was subjected to an enzymatic treatment to improve high-pressure hydrolysis of this raw material; finally, an alcoholic fermentation was carried out with Saccharomyces cerevisiae. The results showed significant time saving by applying a HTP compared to a conventional pretreatment (carried out in autoclaves). A final hydrolysis of $70.4 \%$ of the nopal cladodes (combination of thermal pretreatment and enzymatic hydrolysis) and bioethanol production of $6.6 \mathrm{~g} / \mathrm{L}$ were achieved.
\end{abstract}

\section{INTRODUCCIÓN}

Los problemas ambientales y de salud generados por el uso intensivo de combustibles de origen fósil han estimulado el desarrollo de las energías denominadas alternativas, y varios gobiernos se han planteado metas de sustitución por combustibles renovables a fin de disminuir la dependencia del petróleo (Buruiana et al. 2014, Tacias et al. 2016). Dentro de los diferentes tipos de combustibles renovables destaca el bioetanol, producto que puede sustituir parcial o totalmente a la gasolina en automotores y que ha mostrado un aumento constante en su producción en los últimos años, especialmente en Estados Unidos y Brasil (Soccol et al. 2010, Boucher et al. 2015). El surgimiento del bioetanol y el biodiesel ocurrió a la par que la de sus hermanos de tipo fósil, sin embargo, su desarrollo no ha sido fácil y sólo desde hace algunos años se han considerado como una alternativa viable. Actualmente los biocombustibles se clasifican como combustibles de primera generación en los que se emplea biomasa fácilmente fermentable, con métodos de pretratamiento sencillos que generan subproductos con alto valor agregado (Álvarez 2009, Serna et al. 2010). Entre los cultivos más utilizados para la producción de bioetanol de primera generación se pueden mencionar el maíz, la caña de azúcar, el arroz, el trigo y la cebada.

Como consecuencia, el uso de estos cultivos como fuentes de combustibles ha provocado el aumento de precio y el de sus productos derivados, poniendo en riesgo la seguridad alimentaria de algunos países (de la Torre y He 2007). Aunado a lo anterior, se estima que para sustituir por completo a la gasolina y el diésel con biocombustibles líquidos a nivel mundial, es necesario producir diariamente 30 millones de barriles de bioetanol y 23 millones de biodiesel, lo que implicaría cultivar 15 veces la superficie que actualmente se planta de caña de azúcar, cinco veces la superficie de maíz y 20 veces la superficie de palma de aceite. Las consecuencias de este hipotético escenario serían un aumento en el precio de los cultivos, un impacto económico y social y ambiental, pues sería necesario desarrollar monocultivos, probablemente manipulados genéticamente, disminuyendo la biodiversidad en las áreas donde se desarrollen, además del excesivo consumo de agua y uso de la tierra que no estaría destinado a otros productos para consumo humano (de la Torre y He 2007, HLPE 2013).

Por ello ha surgido la necesidad de producir combustibles renovables con materias primas que no compitan con los cultivos primarios, desarrollando una segunda generación de biocombustibles, donde los esfuerzos se enfoquen a cultivos secundarios y otro tipo de biomasa lignocelulósica (Sims et al. 2010). Algunos de los sustratos más estudiados son el bagazo de caña de azúcar (Cardona et al. 2010), cultivos forestales de rotación corta (álamos, sauces, eucaliptos) (Porzio et al. 2012), hierbas perennes (pasto elefante, pasto varilla y alpiste) (Kallioinen et al. 2012), remolachas (Jiménez et al. 2012), residuos de la industria de la madera, la silvicultura y la agricultura (Jafari et al. 2011, Sarkar et al. 2012, Mood 
et al. 2013, Tayeh et al. 2014, Domínguez-Bocanegra et al. 2015) y hasta desechos de billetes (Sheik et al. 2013). Sin embargo, se han presentado algunos inconvenientes en la obtención de biocombustibles de segunda generación por vías bioquímicas, como son el escaso desarrollo de tecnologías de pretratamiento para la liberación de los azúcares (Naik 2010), el incipiente estudio de subproductos de interés comercial (Feria et al. 2012) y la naturaleza bioquímica de los mismos (Jiménez et al. 2012). También tiene una fuerte influencia el desconocimiento de compuestos que puedan inhibir o retardar las reacciones enzimáticas de hidrólisis (Ximenes et al. 2010).

El nopal (Opuntis spp.) ha sido utilizado como fuente de energía renovable para la producción de biogás, obteniéndose buenos rendimientos (Ramos et al. 2014); además, esta planta presenta bajos costos de mantenimiento y una amplia distribución mundial. Por otra parte, debido a las características de la planta y su rápida regeneración, los productores de tuna $\mathrm{y}$ nopalito realizan podas periódicas generando miles de toneladas de biomasa que no tienen un uso específico, por lo que su deficiente eliminación puede implicar problemas de contaminación y generación de plagas. En 1923 se propuso la utilización de etanol obtenido de Opuntia ficus-indica (OFI) como combustible para aeroplanos (Engineering 1923). Se han desarrollado pocos estudios subsecuentemente: Retamal (1987) produjo etanol a partir de frutos (tuna) y cladodios de Opuntia ficus-indica. En este trabajo se emplearon condiciones de hidrólisis ácidas en el pretratamiento del sustrato, con un tratamiento enzimático (celulasas comerciales) subsiguiente. Santos et al. (2016) y Kuloyo et al. (2014) resaltaron que el éxito de la fermentación alcohólica depende de una mayor hidrólisis de los residuos, la cual estará directamente relacionada con una mayor concentración de azúcares. En general, para la producción de biocombustibles de segunda generación es necesario desarrollar técnicas que ayuden a aprovechar los residuos lignocelulósicos (entre ellos los de Opuntia ficus-indica) con miras a desarrollar una bioindustría que integre operaciones unitarias y procesos biotecnológicos eficientes (Hamdi 1997).

Relacionado con lo anterior, se han desarrollado varios tipos de pretratamientos de la biomasa de origen lignocelulósico con el objetivo de mejorar el acceso a las fibras de celulosa y hemicelulosa y mejorar el tratamiento enzimático. Amiri y Karimi (2015) desarrollaron un proceso de autohidrólisis de residuos lignocelulósicos, obteniendo mayores rendimientos de oligosacáridos y bajas concentraciones de compuestos como furfural, 5-hidroximetilfurfural (HMF) y ácido acético. Taherzadeh y Karimi (2007) y Zhu et al. (2015) aplicaron el concepto de autohidrólisis complementado con la adición de bajas concentraciones de ácido (0.07-1.5\%). Estos autores observaron un aumento en la concentración de carbohidratos libres, además de que el residuo resultante fue más susceptible de ser hidrolizado por métodos enzimáticos. Por otra parte, Vargas y Pereira (2010) encontraron que en el pretratamiento hidrotérmico de residuos ligniocelulósicos el principal factor que influye en la liberación de monosacáridos es la presión.

En este trabajo se planteó como objetivo desarrollar una metodología que mejore la liberación de los carbohidratos presentes en los cladodios de OFI para que puedan ser utilizados como sustrato en la producción de etanol de segunda generación, además de generar una alternativa que pueda utilizarse para el aprovechamiento de los residuos de poda en cultivares de OFI y la disminución de su impacto contaminante. Con lo anterior, se pretende contribuir a disminuir la emisión de gases de efecto invernadero. Como primera etapa se analizó el efecto del pretratamiento hidrotérmico $(\mathrm{PTH})$; posteriormente se realizó de un tratamiento enzimático utilizando celulasas y xilanasas comerciales, y se analizó la liberación de azúcares de cladodios de O. ficus-indica; finalmente, los hidrolizados fueron sometidos a fermentación alcohólica con Sacharomyces cereviceae. Las variables que se analizaron para el PTH fueron temperatura, concentración de ácido y tiempo de residencia, todo a presión constante de $10 \mathrm{~atm}$.

\section{MATERIALES Y MÉTODOS}

\section{Materia prima}

Se trabajó con un lote de cladodios jóvenes con edad promedio de 3 meses (brotes de alrededor de 18 a $25 \mathrm{~cm}$ de longitud) de Opuntia ficus-indica obtenidos de un vivero en el estado de Hidalgo, México $\left(19^{\circ} 41^{\prime} 50.4^{\prime \prime} \mathrm{N}, 98^{\circ} 47^{\prime} 20.5^{\prime}\right.$ 'W) en septiembre de 2015. Los cladodios fueron segmentados en cubos de $1 \mathrm{~cm}^{3}$, los cuales se deshidrataron a una temperatura de $60{ }^{\circ} \mathrm{C}$. La muestra deshidratada se molió y se cribó en una malla del número 60 (tamaño de partícula de $250 \mu \mathrm{m}$ ). La harina obtenida se guardó en bolsas plásticas que fueron almacenadas en un lugar fresco y seco. Los análisis fisicoquímicos realizados a esta muestra fueron los siguientes: determinación de humedad, determinación de extracto étereo por el método de Soxhlet, cenizas (AOAC 2016), lignina (TAPPI 2009), celulosa (Browning 1967) y el porcentaje de mucílago como parte de los compuestos solubles en agua (Rodríguez et al. 2011). 


\section{Pretratamiento hidrotérmico (PTH)}

La harina de nopal deshidratada fue resuspendida en agua en una relación líquido-sólido (L:S) de 15 (agua:harina, $\mathrm{mL} / \mathrm{g}$ ). Para el tratamiento hidrotérmico se empleó un reactor de acero inoxidable de alta presión (RAP) (Prendo RAP-2000 ${ }^{\mathrm{TM}}$ ) de 2 L. Se llevó a cabo un primer PTH con el fin de determinar la temperatura donde se liberarán mas azúcares, se utilizó sólo agua y se ensayaron las siguientes temperaturas: $150,175,185$ y $205^{\circ} \mathrm{C}$ durante $5 \mathrm{~min}$. Una vez determinada la temperatura en la que se liberó la mayor cantidad de azucares reductores totales (ART), se procedió a analizar el efecto combinado del $\mathrm{H}_{2} \mathrm{SO}_{4}$ $(0.5,1,1.5$ y $2 \%)$ y la temperatura sobre la liberación de ART a diferentes tiempos de reacción (1, 5 y $15 \mathrm{~min}$ ). Al final de cada tratamiento, las muestras obtenidas fueron filtradas con papel Whatman núm. 1 y lavadas con agua destilada. El residuo sólido se secó a $60{ }^{\circ} \mathrm{C}$ y se mantuvo en un lugar fresco y seco hasta su uso.

\section{Tratamiento enzimático}

El residuo fue sometido a un tratamiento enzimático con la enzima $\beta$-glucanasa (Sigma-Aldrich, G4423), para lo cual se midió la actividad endo- $\beta-1,4-$ glucanasa de acuerdo con Ghose (1987). Las actividades enzimáticas de este catalizador se expresaron en unidades internacionales (UI), donde una unidad de actividad se definió como la cantidad de enzima capaz de liberar $1 \mu \mathrm{mol}$ de azúcar (equivalente a glucosa) por minuto. Se ensayaron 1, 5, 10, 20 y 40 UI, a una relación L:S de 15 en buffer de acetatos $(100 \mathrm{mM}, \mathrm{pH} 4.8)$ a $40{ }^{\circ} \mathrm{C}$ y $175 \mathrm{rpm}$. Al final del tratamiento se realizó una filtración para obtener la fracción líquida con los ART obtenidos.

\section{Fermentación alcohólica}

Con los azúcares obtenidos en el pretratamiento hidrotérmico con ácido diluido y en el tratamiento enzimático, se llevó a cabo una fermentación a $30^{\circ} \mathrm{C}$ con $S$. cereviceae obtenida del cepario del Laboratorio de Agrobiotecnología de la Universidad Politécnica de Pachuca, la cual fue aislada de residuos de nopal. El inóculo inicial fue de $1 \times 10^{6}$ células $/ \mathrm{mL}$, sin agitación y con un $\mathrm{pH}$ de 4.5. Esta levadura previamente fue activada por cultivo en caldo YPD $\left(\right.$ Bioxon $^{\mathrm{TM}}$ ), incubada a $30^{\circ} \mathrm{C}, 150 \mathrm{rpm}$, durante $24 \mathrm{~h}$.

\section{Métodos analíticos y análisis estadísticos}

Para la determinación de los ART se empleó la técnica propuesta por Miller (1959), utilizando una longitud de onda de $525 \mathrm{~nm}$ (Biochrom Libra $\left.\mathrm{S} 11^{\mathrm{TM}}\right)$. Para la caracterización de los azúcares se usó un equipo HPLC (Thermo Scientific Ultimate 3000) acoplado a un detector de índice de refracción (Refractomax 520 ${ }^{\mathrm{TM}}$ ) con la columna Rezex ${ }^{\mathrm{TM}} \mathrm{RPM}-$ Monosaccharide $\mathrm{Pb}^{+2} 8 \%$, a una velocidad de flujo de $0.6 \mathrm{~mL} / \mathrm{min}$ y agua como fase móvil. Para determinar el etanol se utilizó un cromatografo de gases (Thermo Scientific Trace $1310^{\mathrm{TM}}$ ) con un detector de ionización de flama (FID) acoplado a una columna zebron ZB-Bioethanol ${ }^{\mathrm{TM}}$ de $15 \mathrm{~m}$ de longitud. Las condiciones de inyección fueron las siguientes: temperatura de $250{ }^{\circ} \mathrm{C}$; temperatura del detector FID, $350{ }^{\circ} \mathrm{C}$, y $1.5 \mathrm{~mL} / \mathrm{min}$ de Helio como gas acarreador. Las muestras fueron previamente filtradas con membranas de $0.22 \mu \mathrm{m}$. Se utilizó una rampa de temperatura de 45 a $250{ }^{\circ} \mathrm{C}$ con incrementos de $5^{\circ} \mathrm{C}$. Todas las pruebas se trabajaron por triplicado. Se realizó un analisis de varianza y una comparación entre medias con el programa estadístico SigmaPlot ${ }^{\mathrm{TM}}$ v. 11.0.

\section{RESULTADOS Y DISCUSIÓN}

\section{Composición de la materia prima}

Los resultados de los análisis fisicoquímicos realizados a los cladodios de OFI se presentan en el cuadro I. Como características principales se observan alto contenido de agua $(94.7 \%)$, bajo contenido de lignina $(2.65 \%)$ y alta concentración de compuestos solubles en agua (63\%). En estas condiciones se estimó una relación L:S de alrededor de 19 (94.735:5.265), valor que puede compararse con lo descrito en otros trabajos (Ginestra et al. 2009, Yang et al. 2015). El alto porcentaje de humedad es típico de plantas con metabolismo CAM, pues producen hidrocoloides que permiten almacenar agua en los tejidos de estos vegetales (Sáenz et al. 2004). Esta característica puede tener una ventaja a nivel industrial, ya que representaría un ahorro considerable de agua si se realizara una hidrólisis química de los cladodios.

Las variaciones observadas en la caracterización fisicoquímica puede ser resultado de factores edáficos de los sitios de cultivo, la época de cultivo y la edad de la planta (Stintzing y Carle 2005, Ribeiro et al. 2010).

\section{Comparación del PTH sin ácido y con ácido di- luido}

En la figura 1a se presenta el efecto del tiempo sobre la liberación de los ART por acción del PTH empleando sólo agua. En esta primera prueba se fijó una temperatura de $175^{\circ} \mathrm{C}$, la cual es cercana a la empleada en el PTH de biomasa (Chen et al. 2014, 
CUADRO I. CARACTERÍSTICAS FISICAS Y QUÍMICAS DE CLADODIOS DE Opuntia ficus-indica*

\begin{tabular}{lcccccc}
\hline Autor & $\begin{array}{c}\text { Humedad } \\
(\%)\end{array}$ & $\begin{array}{c}\text { Extracto etéreo } \\
(\%)\end{array}$ & $\begin{array}{c}\text { Cenizas } \\
(\%)\end{array}$ & $\begin{array}{c}\text { Lignina soluble } \\
\text { e insoluble }(\%)\end{array}$ & $\begin{array}{c}\text { Solubles en } \\
\text { agua }(\%)\end{array}$ & $\begin{array}{c}\text { Celulosa } \\
(\%)\end{array}$ \\
\hline En este trabajo & $94.735( \pm 0.21 \%)$ & $3.897( \pm 0.365)$ & $15( \pm 0.146)$ & $2.65( \pm 0.115)$ & $63.077( \pm 1.571)$ & $18.876( \pm 0.565)$ \\
Malainine et al. 2003 & N.R. & 7.2 & 19.6 & 3.6 & 78 & 21.6 \\
Kuloyo et al. 2014 & $88-95$ & N.R. & 16.8 & 12.3 & 24.3 & 23.1 \\
Yang et al. 2015 & 93.9 & N.R. & 23.7 & 16 & 25.0 & 17.7 \\
Ginestra et al. 2009 & N.R. & 0.63 & n.r & 13.1 & 13.5 \\
\hline
\end{tabular}

*Los resultados se presentan en base seca, a excepción de la humedad N.R.: no reportado
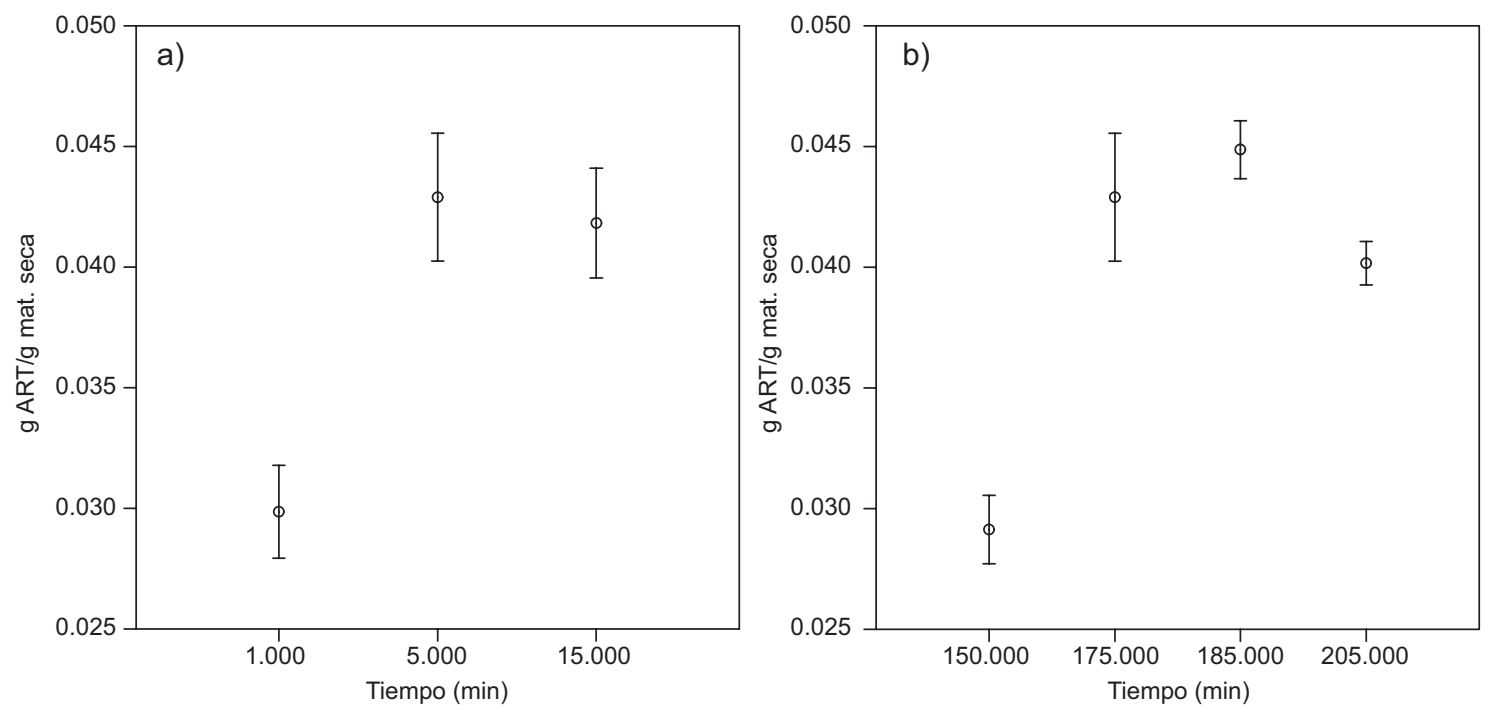

Fig. 1. (a) Liberación de ART en la fase líquida en un PTH empleando sólo agua a diferentes tiempos de cocción. (b) ART liberados a diferentes temperaturas con un tiempo fijo de 5 min

Gonçalves et al. 2015, Vallejos et al. 2015). Como se puede observar, entre los tiempos de tratamiento de 5 y 15 min no se detectó una diferencia significativa, por lo cual se eligió el tiempo de $5 \mathrm{~min}$ (por ahorro de energía) para estudiar el efecto de la temperatura sobre la liberación de ART. Las temperaturas probadas fueron de $150,175,185$ y $205^{\circ} \mathrm{C}$ (Fig. 1b). La mayor liberación de ART en el intervalo de temperatura estudiado se detectó entre $175 \mathrm{y}-185^{\circ} \mathrm{C}$ con 0.0429 y $0.0449 \mathrm{~g} \mathrm{ART} / \mathrm{g}$ materia seca respectivamente, no observándose diferencias significativas entre ambas condiciones.

A $150{ }^{\circ} \mathrm{C}$ se presentó una menor liberación de azúcares, debido posiblemente a que hay un porcentaje muy bajo de hidrólisis de la celulosa. Este comportamiento se ha observado con otros sustratos y probablemente se deba a que no se reúne la energía suficiente para ionizar el agua del medio $\left(\mathrm{H}^{+}\right)$y liberar el ácido acético de las cadenas de este biopolímero (Carvalheiro et al. 2009). Por otra parte, se observó que a $205^{\circ} \mathrm{C}$ también se tienen bajas cantidades de ART, resultado que puede deberse a que a temperaturas más elevadas, los azúcares comienzan un proceso de degradación por pirolisis, con la consecuente formación de furfurales, $\mathrm{CO}_{2}$ y $\mathrm{H}_{2} \mathrm{O}$ (Baêta et al. 2016).

Al comparar los resultados entre el grupo con temperaturas de 175 y $185^{\circ} \mathrm{C}$, no se encontró diferencia significativa $(\mathrm{p}<0.05)$, por lo que se fijó $175^{\circ} \mathrm{C}$ como la temperatura de proceso. A continuación se estudió la influencia del ácido sulfúrico $(0$, $0.5 \%, 1 \%, 1.5 \%$ y $2 \%$ ) y el tiempo de reacción (1, 5 y 15 min) sobre la liberación de ART (Fig. 2).

De acuerdo con los resultados obtenidos, se concluye que al utilizar sólo agua en el PTH se liberan menos de $0.05 \mathrm{~g}$ ART $/ \mathrm{g}$ de materia seca. Y en un PTH con ácido sulfúrico diluido se demuestra que, 


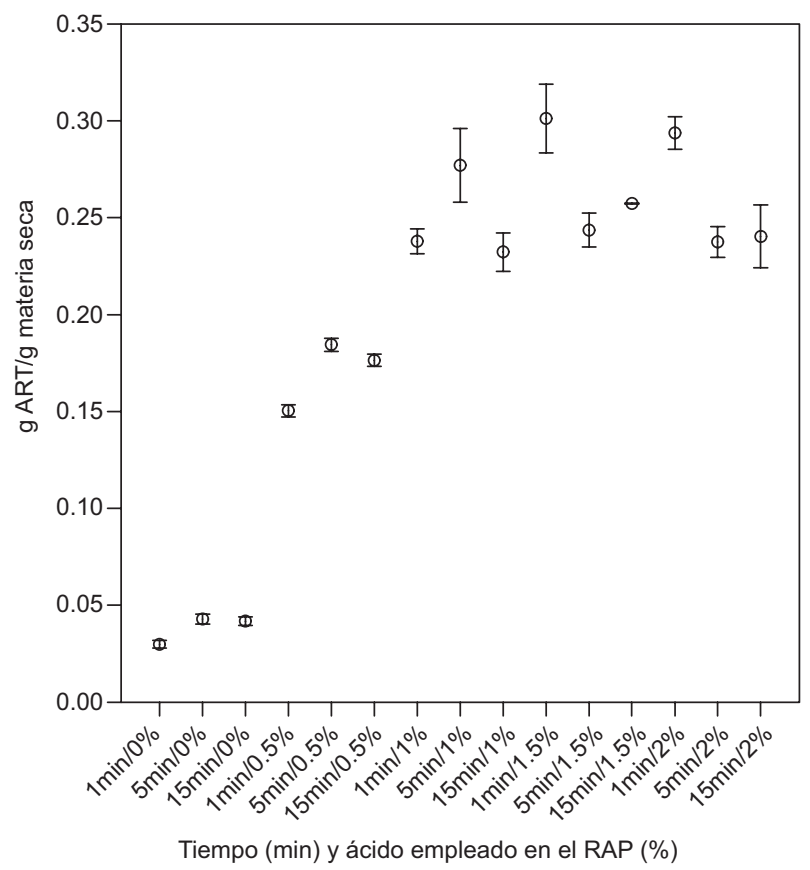

Fig. 2. ART liberados con diferentes porcentajes de $\mathrm{H}_{2} \mathrm{SO}_{4} \mathrm{y}$ tiempos en un RAP a $175^{\circ} \mathrm{C}$

independientemente del tiempo y la temperatura, la liberación de ART aumenta en aproximadamente $336 \%$ en el tratamiento con $0.5 \%$ de ácido, $480 \%$ en el tratamiento con $1 \%$ de ácido y $520 \%$ en los tratamientos con 1.5 y $2 \%$ de ácido. Otros autores han obtenido resultados similares, observando que al adicionar más ácido se tiene un aumento en la concentración de monosacáridos (Boucher et al. 2015, Kim et al. 2016). Es posible que este efecto esté relacionado con una hidrolisis de las cadenas de polisacáridos con el consecuente aumento de monosacáridos. Por otra parte, el aumento en la liberación de ART se puede deber al hecho de que al adicionar ácido a la fase acuosa aumenta la concentración de iones $\mathrm{H}^{+}$que no solamente hidrolizan la celulosa, sino también las cadenas de oligosacáridos que se van desprendiendo, lo cual no ocurre si sólo se aplica un pretratamiento con agua.

Por otra parte, el tiempo y la concentración de ácido también mostraron un efecto muy importante sobre la liberación de ART: a 1 min con $2 \% \mathrm{de}_{2} \mathrm{SO}_{4}$ se obtuvo, en promedio, $0.294 \mathrm{~g}$ ART/g de materia seca, pero a los $15 \mathrm{~min}$ hay una disminución de hasta $0.240 \mathrm{~g} \mathrm{ART} / \mathrm{g}$ de materia seca. Es importante señalar que un tratamiento ácido prolongado tiene un efecto negativo en la concentración de ART, ya que a ciertas condiciones de temperatura y alta acidez los azúcares se comienzan a degradar a furfurales
(Ojumu y Ogunkunle 2005). Este efecto también se observó en los tratamientos de 5 y 15 min con 1.5 y $2 \%$ de $\mathrm{H}_{2} \mathrm{SO}_{4}$, donde se obtuvieron $0.25,0.26$ y 0.27 g ART/g de materia seca, respectivamente (Fig. 2).

Al final de esta etapa se observó que $175^{\circ} \mathrm{C}, 1 \%$ de $\mathrm{H}_{2} \mathrm{SO}_{4}$ y 5 min fueron las condiciones de mayor porcentaje de hidrólisis, ya que con ellas se obtuvo la mayor cantidad de ART por gramo de harina de nopal pretratada. El análisis estadístico no indicó diferencias significativas entre los otros tratamientos $(p<0.05)$. Se procedio a evaluar el efecto de la concentración de ácido sobre la cantidad de materia hidrolizada (Fig. 3); los resultados obtenidos señalan que con un $\mathrm{PTH}$ sin la adición de $\mathrm{H}_{2} \mathrm{SO}_{4}$ se tiene en promedio $64.36 \%$ de materia hidrolizada y el resto son residuos sólidos (35.64\%). El porcentaje de hidrólisis de la harina aumenta conforme se incrementa la concentración de ácido sulfúrico; sin embargo, como ya se ha mencionado, a mayor exposición de la materia prima a condiciones ácidas, se corre el riesgo de menor rendimiento de los azúcares. Este resultado suguiere que primeramente hay una hidrolisis del mucílago y compuestos solubles y parte de la celulosa amorfa, y el residuo resultante es la celulosa cristalina que necesita de condiciones de hidrólisis mas severas para liberar monosacáridos. A medida que se agrega ácido, el porcentaje de materia hidrolizada aumenta hasta cierto punto, al igual que los ART liberados en la fase líquida, ya que como se indicó anteriormente,

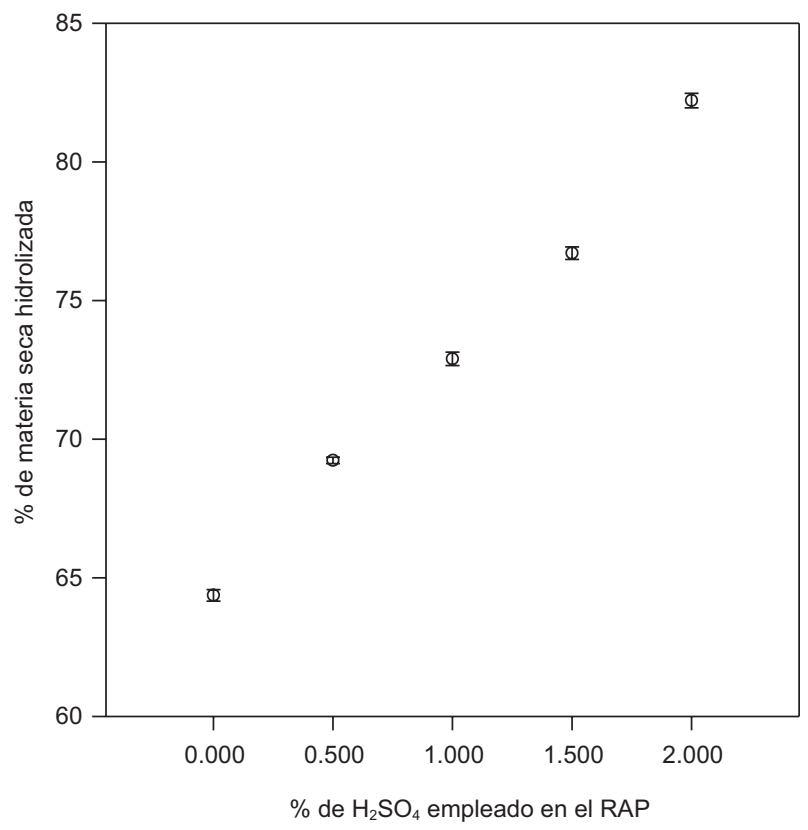

Fig. 3. Porcentajes de materia hidrolizada con PTH con y sin ácido diluido a $175^{\circ} \mathrm{C}$ y 5 min de reacción 
una mayor concentración de iones $\mathrm{H}^{+}$parece tener un mayor efecto positivo sobre la hidrólisis de las cadenas de polisacáridos presentes en los cladodios que cuando se emplea sólo agua. Para la siguiente etapa de este trabajo y con las condiciones de hidrólisis ácida elegidas ( $1 \%$ de $\mathrm{H}_{2} \mathrm{SO}_{4}$ y 5 min de tratamiento a $175^{\circ} \mathrm{C}$ ), se observó que queda un residuo sólido que representa el $27.11 \%$ del material lignocelulósico sin hidrolizar. Este material fue sometido a un tratamiento enzimático para aumentar el rendimiento de los azúcares liberados.

Respecto a la fracción acuosa, se determinó el perfil de azúcares por cromatografía líquida y se observó que alrededor de un $57.14 \%$ correspondió a galactosa, seguido de arabinosa $(20.51 \%)$, glucosa $(13.18 \%)$ y xilosa $(9.17 \%)$. Estos azúcares están presentes en las cadenas de polisacáridos que componen los mucílagos de nopal (Domínguez 1995), que al hidrolizarse se liberaron al medio.

Por otra parte, una de las ventajas que se tienen al emplear un RAP para aplicar el PTH y el PTH con ácido en harina de OFI, es el ahorro significativo de tiempo y ácido, ya que en condiciones de $121^{\circ} \mathrm{C}$ y $1 \mathrm{~atm}$ de presión durante $27.25 \mathrm{~min}$ con $3.47 \%$ de $\mathrm{H}_{2} \mathrm{SO}_{4}$ (datos sin publicar) con esta misma materia prima. Al comparar este resultado con trabajos similares para tratamientos de OFI, se tiene que se han empleado 50 min y $1.5 \%$ de $\mathrm{H}_{2} \mathrm{SO}_{4}$ (Kuloyo et al. 2014), 60 min y $1.25 \%$ de $\mathrm{H}_{2} \mathrm{SO}_{4}$ (Santos et al. 2016) y 30 min con $3.65 \%$ de $\mathrm{HCl}$ (Retamal 1987).

\section{Tratamiento enzimático}

El residuo sólido resultante del PHT fue sometido a un tratamiento enzimático para determinar su efecto sobre la liberación de azúcares. Los resultados obtenidos mostraron que se obtuvo un hidrolizado final que representó el $70.4 \%$ del total del sustrato empleado (1.66 g) después de $36 \mathrm{~h}$ de tratamiento (Fig. 4), resultado similar al obtenido por Arantes y Saddler (2011). En este proceso se utilizó una cantidad de enzima de $200 \mathrm{mg} / \mathrm{g}$ sustrato, mientras que dichos autores utilizaron $63 \mathrm{mg} / \mathrm{g}$ sustrato. El hecho de haber utilizado mas enzima se puede deber a varios factores, como pueden ser: a) una afinidad diferente de la enzima por sustrato; b) una fuente diferente de la enzima utilizada; c) factores de inhibición por sustrato debido a que la complejidad del hidrolizado en cuanto a su composición de azúcares es muy alta; o d) la presencia de compuestos inhibitorios en el residuo, propios de OFI, que inhiben la acción de la celulasa, tal como lo describe Ximenes et al. (2010).

El análisis por HPLC, mostró principalmente la presencia de glucosa, fructosa y xilosa a una

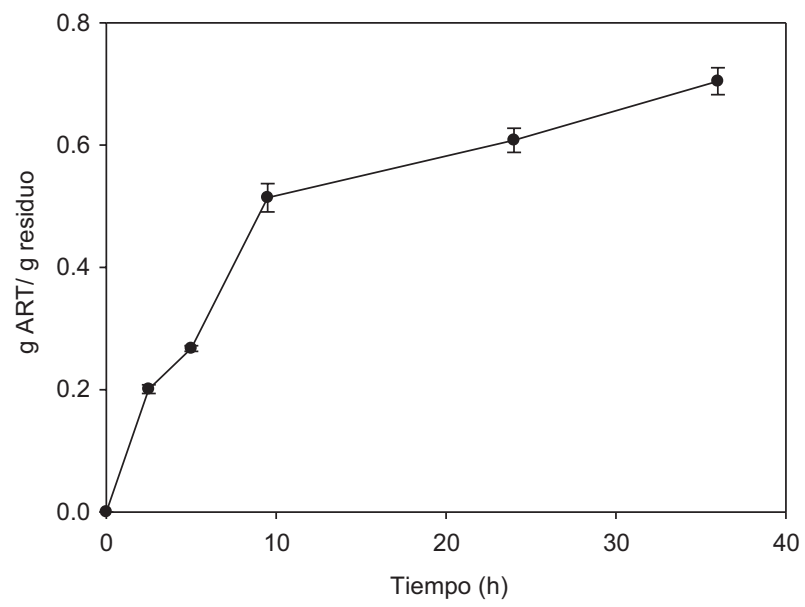

Fig. 4. Obtención de ART mediante un tratamiento enzimático

concentración de 52.32, 3.47 y 1.73 g/L que representa el $89.54,5.95$ y $2.96 \%$, respectivamente. En menor concentración se detectó galactosa y arabinosa. Es probable que la reacción que ocurre en las condiciones ensayadas favorezca el rompimiento de los enlaces endo- $\beta-1,4$ de la celulosa; asimismo, es posible que el PTH con ácido diluido genere fibra reactiva que es más susceptible a un tratamiento enzimático. Se han observado resultados similares con residuos lignocelulósicos de otro origen en los cuales se ve favorecida la hidrólisis enzimática al recibir un PTH con o sin ácido agregado (Taherzadeh y Karimi 2008, Amiri y Karimi 2015, Gonçalves et al. 2015).

\section{Fermentación alcohólica}

La fermentación alcohólica se llevó a cabo con $S$. cerevisiae durante 72 h (Fig. 5a), con una concentración inicial de azúcares de $21.269 \mathrm{~g} / \mathrm{L}$. El balance estequiométrico sugiere un rendimiento teórico de $10.87 \mathrm{~g} / \mathrm{L}$ de etanol si todos los azúcares son glucosa y/o fermentables. Al cabo del periodo señalado se produjeron alrededor de $6.681 \mathrm{~g} / \mathrm{L}$ de etanol, es decir, un rendimiento real del $61.46 \%$. Por otra parte, el azúcar residual fue de $4.295 \mathrm{~g} / \mathrm{L}$, lo que generó un consumo de azúcares del 77 \% que es muy cercano a la concentración de glucosa y fructosa detectadas en el proceso de hidrólisis, que además fueron los azúcares mayoritariamente consumidos. Se observó que los azúcares como galactosa, xilosa y arabinosa no fueron asimilados por la levadura empleada (Fig. 5b).

Son pocos los trabajos en que se ha estudiado la posibilidad de producir etanol a partir de azúcares obtenidos de OFI. Cabe resaltar que en casi todos estos trabajos se ha empleado $S$. cerevisiae como organismo fermentador. Por otro lado, a fin de comparar 

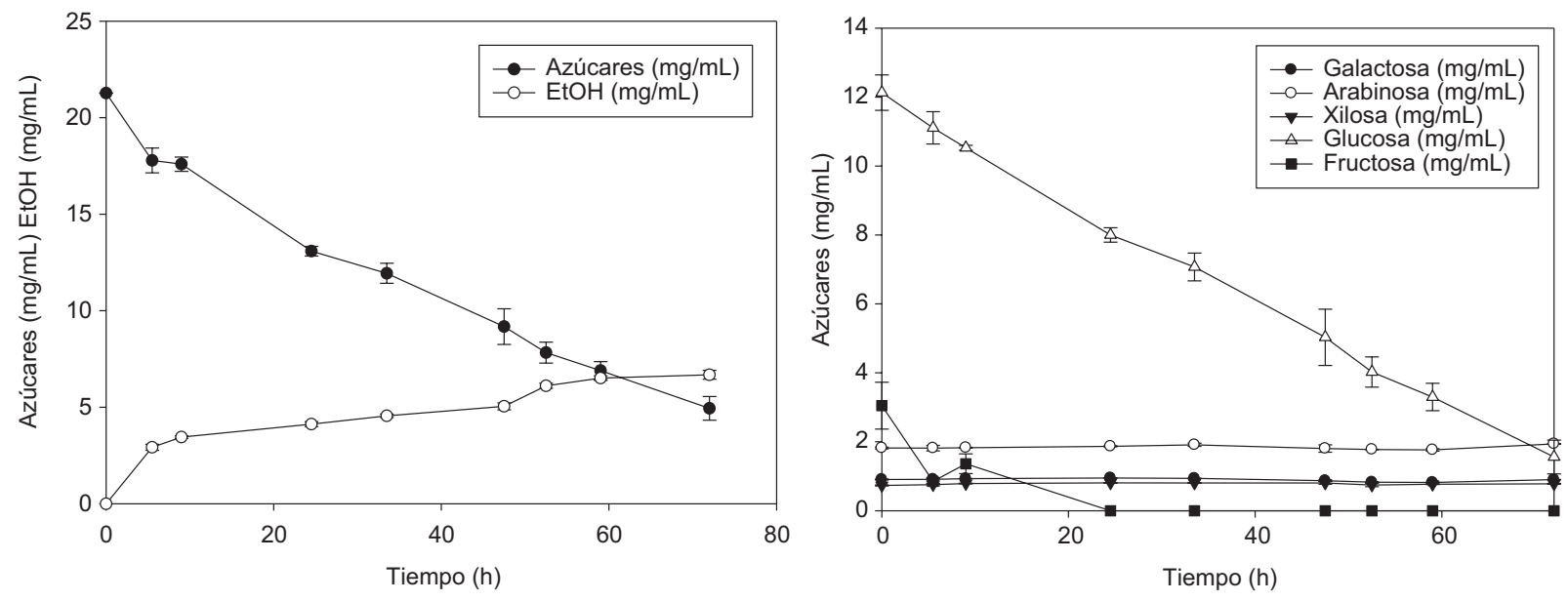

Fig. 5. (a) Producción de etanol a partir de los azúcares obtenidos en el PTH con ácido diluido y con el tratamiento enzimático al residuo sólido. (b) Consumo de los azúcares durante la fermentación alcohólica

los resultados obtenidos es muy importante conocer la relación sólido-líquido de la cual se partió en cada trabajo, pues esta variable gobierna directamente la liberación de azúcares e indirectamente la producción de alcohol. Retamal et al. (1987) observaron que en un pretratamiento con ácido clorhídrico y uno enzimático de cladodios de Opuntia se obtenía hasta $17.7 \mathrm{~g} / \mathrm{L}$ de etanol; sin embargo, no se especifica la relación sólido-líquido utilizada. En un trabajo similar, Kuloyo et al. (2014) probaron una relación sólido-líquido de 3.36 , produciendo $25.8 \mathrm{~g} / \mathrm{L}$ de etanol. Al comparar este resultado con el obtenido en el presente trabajo, se observa que al haber mayor concentración de azúcares en el medio de producción (hidrolizados de Opuntia), se pueden obtener mayores rendimientos. Por otra parte, Santos et al. (2016), produjeron con hidrolizados de Opuntia, $3.5 \mathrm{~g} / \mathrm{L}$ de etanol con una relación S:L de 13.33, lo que sugiere que independientemente de la relación S:L empleada, la complejidad en la composición de carbohidratos del hidrolizado es un factor que impide obtener altos rendimientos de etanol. Un factor a tomar en cuenta al utilizar PTH para hidrolizar cladodios de Opuntia spp. es la generación de xilooligosacáridos, que tienen gran importancia en las industrias alimenticia y farmacéutica, principalmente (Gullón et al. 2011, Xiao et al. 2013). Esta puede ser una alternativa atractiva de uso del hidrolizado.

En general, los rendimientos de alcohol conseguidos en esta investigación imponen otro tipo de retos debido a la complejidad del hidrolizado obtenido, ya que se requieren microorganismos altamente especializados capaces de fermentar las pentosas y hexosas presentes. Stintzing y Carle (2005) desarrollaron productos de Opuntia spp. enfocados a la industria farmacéutica, cosmética y alimentaria, ya que esta planta es un fuente potencial de otros productos con interés biotecnológico, que van de pectinas (Goycoolea y Cárdenas 2003), fibras solubles (Sáenz y Sepúlveda 2001), compuestos antioxidantes y fibra insoluble, a compuestos con propiedades anticancerígenas (Serra et al. 2013), entre otros.

Se le han atribuido varios beneficios a esta planta, muchos de los cuales se pueden derivar de las cadenas de oligosacáridos que se liberan durante un PTH, como los xilooligasacáridos, que recientemente han tenido aplicaciones en las industrias antes mencionadas (Gullón et al. 2011, Xiao et al. 2013). Aunado a lo anterior, el residuo sólido, compuesto principalmente por celulosa (del cual se obtiene una importante cantidad de glucosa), podría utilizarse para la producción de etanol y — a reserva de analizar su factibilidadconcentrar estos azúcares para manejar porcentajes más elevados en una fermentación alcohólica.

\section{CONCLUSIONES}

Con la metodología generada se alcanzó un $70.4 \%$ de hidrólisis de los cladodios de OFI empleando 5 min de proceso. Un PTH sobre OFI utilizando únicamente agua (autohidrólisis) produjo bajos rendimientos de ART $(0.05 \mathrm{~g} / \mathrm{L})$ y altos rendimientos de oligosacáridos. Por ello, para mejorar la liberación de monosacáridos es necesario agregar ácido a una concentración de $1 \%$. Además, el tiempo y el gasto de ácido disminuyen al emplear un PTH sobre este sustrato. Por otro lado, la hidrólisis enzimática del residuo sólido resultante incrementó la liberación de 
azúcares, liberando glucosa como principal azúcar, lo que podría mejorar la producción de etanol. Sin embargo, la complejidad del hidrolizado en cuanto a su composición química tuvo como principal efecto una baja producción de etanol si se compara con lo obtenido con otro tipo de residuos estudiados. Por lo anterior se requiere integrar a este proceso microorganismos capaces de metabolizar no sólo glucosa, sino tambíen otro tipo de azúcares como pentosas, o utilizar microorganismos genéticamente modificados capaces de fermentar los azúcares presentes en el hidrolizado para hacer rentable la producción de bioetanol a partir de cladodios de Opuntia spp.

\section{AGRADECIMIENTOS}

Este trabajo fue realizado gracias al financiamiento del proyecto número 195157 del Fondo Sectorial Secretaría de Agricultura, Ganadería, Desarrollo Rural, Pesca y Alimentación-Consejo Nacional de Ciencia y Tecnología (SAGARPA-CONACyT) de México.

\section{REFERENCIAS}

Álvarez M.C. (2009). Biocombustibles: desarrollo histórico-tecnológico, mercados actuales y comercio internacional. Economía Informa 359, 63-89.

Amiri H. y Karimi K. (2015). Autohydrolysis: A promising pretreatment for the improvement of acetone, butanol, and ethanol production from woody materials. Chem. Eng. Sci. 137, 722-729.

DOI: $10.1016 /$ j.ces.2015.07.020

AOAC (2016). Moisture and volatile matter (926.12). 20a ed. Association of Official Analytical Chemists, Washington, D.C., EUA, 957 pp.

Arantes V. y Saddler J.N. (2011). Cellulose accessibility limits the effectiveness of minimum cellulase loading on the efficient hydrolysis of pretreated lignocellulosic substrates. Biotechnol. Biofuels 4 (1), 3. DOI: 10.1186/1754-6834-4-3

Baêta L.B.E., Sousa L.D.R., Herrera A.O.F., Alves G.L.V. y de Aquino S.F. (2016). Optimization of sugarcane bagasse autohydrolysis for methane production from hemicellulose hydrolyzates in a biorefinery concept. Bioresour. Technol. 200, 137-146.

DOI: 10.1016/j.biortech.2015.10.003

Boucher J., Chirat C. y Lachenal D. (2015). Comparison between acid hydrolysis and two-step autohydrolysis for hemicellulosic ethanol production. Cellulose Chem. Technol. 49 (3-4), 303-308
Browning B.L. (1967). Holocellulose preparations. In: Methods of wood chemistry. 1a ed. Interscience Publishers, Nueva York, EUA, 882 pp.

Buruiana C.T., Vizireanu C., Garrote G. y Parajó J.C. (2014). Optimization of corn stover biorefinery for coproduction of oligomers and second generation bioethanol using non-isothermal autohydrolysis. Ind. Crops Prod. 54, 32-39.

DOI: $10.1016 /$ j.indcrop.2014.01.003

Cardona C.A., Quintero J.A. y Paz I.C. (2010). Production of bioethanol from sugarcane bagasse: Status and perspectives. Bioresour. Technol. 101 (13), 4754-4766. DOI: 10.1016/j.biortech.2009.10.097

Carvalheiro F., Silva F.T., Duarte L.C. y Gírio F.M. (2009). Wheat straw autohydrolysis: Process optimization and products characterization. Appl. Biochem. Biotechnol. 153 (1-3), 84-93.

DOI: $10.1007 / \mathrm{s} 12010-008-8448-0$

Chen M.H., Bowman M.J., Dien B.S., Raush K.D., Tumbleson M.E. y Singh V. (2014). Autohydrolysis of Miscanthus giganteus for the production of xylooligosaccharides (XOS): Kinetics, characterization and recovery. Bioresour. Technol. 155, 359-365.

DOI: 10.1016/j.biortech.2013.12.050

De la Torre U.D. y He L. (2007). Is the expansion of biofuels at odds with the food security of developing countries? Biofuels Bioprod. Bioref. 1 (2), 92-102. DOI: $10.1002 / b b b .16$

Domínguez-Bocanegra A.R., Torres-Muñoz J.A. y López R.L. (2015). Production of bioethanol from agroindustrial wastes. Fuel 149, 85-89.

DOI: 10.1016/j.fuel.2014.09.062

Domínguez L.A. (1995). Revisión bibliográfica sobre algunos aspectos tecnológicos y usos alternativos de los cladodios y frutos del nopal (Opuntia spp.) en la alimentación humana. Ciencia Ergo-Sum 2 (2), 231-238.

Engineering (1923). The argus (Melbourne, Vic.: 18481957) [en línea]. https://trove.nla.gov.au/newspaper/ article/1871302 27/12/2017.

Feria M.J., García J.C., Díaz M.J., Fernández M. y López F. (2012). Biorefinery process for production of paper and oligomers from Leucaena leucocephala K360 with or without prior autohydrolysis. Bioresour. Technol. 126, 64-70. DOI: 10.1016/j.biortech.2012.09.051

Ghose T.K. (1987) Measurement of cellulase activities. Pure Appl. Chem. 9 (2), 257-268.

Ginestra G., Parker M.L., Bennett R.N., Robertson J., Mandalari G., Narbad A., Curto R.B.L., Bisignano G., Faulds C.B. y Waldron K.W. (2009). Anatomical, chemical, and biochemical characterization of cladodes from prickly pear [Opuntia ficus-indica (L.) Mill.]. J. Agric. Food Chem. 57 (21), 10323-10330.

DOI: $10.1021 / \mathrm{jf} 9022096$ 
Gonçalves F.A., Ruiz H.A., dos Santos E.S., Teixeira J.A. y de Macedo G.R. (2015). Bioethanol production from coconuts and cactus pretreated by autohydrolysis. Ind. Crops Prod. 77, 1-12.

DOI: $10.1016 / \mathrm{j}$.indcrop.2015.06.041

Goycoolea F.M. y Cárdenas A. (2003). Pectins from Opuntia spp.: A short review. J. Prof. Assoc. Cactus 1, 17-29.

Gullón P., González M.M.J. y Parajó J.C. (2011). Manufacture and prebiotic potential of oligosaccharides derived from industrial solid wastes. Bioresour. Technol. 102 (10), 6112-6119. DOI: 10.1016/j.biortech.2011.02.059

Hamdi M. (1997). Prickly pear cladodes and fruits as a potential raw material for the bioindustries. Bioprocess Eng. 17 (6), 387-391.

DOI: $10.1007 / \mathrm{s} 004490050402$

HLPE (2013). Biofuels and food security. A report by high level panel of experts on food security and nutrition [en línea]. http://www.fao.org/fileadmin/user_upload/hlpe/ hlpe_documents/HLPE_Reports/HLPE-Report-5_Biofuels_and_food_security.pdf 27/11/2017.

Jafari V., Labafzadeh S.R., Jeihanipour A., Karimi K. y Taherzadeh M.J. (2011). Construction and demolition lignocellulosic wastes to bioethanol. Renew. Energy. 36 (11), 2771-2775. DOI: 10.1016/j.renene.2011.04.028

Jiménez I.D., Abreu C.A., López L.V.E., Téllez-Jurado A. y Gracida R.J.N. (2012). Obtención de azúcares fermentables mediante hidrólisis ácida de Beta vulgaris L. Rev. Int. Contam. Ambie. 28 (2), 151-158.

Kallioinen A., Uusitalo J., Pahkala K., Kontturi M., Viikari L., von Weymarn N. y Siika-Aho M. (2012). Reed canary grass as a feedstock for 2nd generation bioethanol production. Bioresour. Technol. 123, 669-672. DOI: 10.1016/j.biortech.2012.07.023

Kim T.H., Ryu H.J. y Oh K.K. (2016). Low acid hydrothermal fractionation of Giant miscanthus for production of xylose-rich hydrolysate and furfural. Bioresour. Technol. 218, 367-372.

DOI: $10.1016 /$ j.biortech.2016.06.106

Kuloyo O.O., du Preez J.C., García A.M del P., Kilian S. G., Steyn L. y Görgens J. (2014). Opuntia ficusindica cladodes as feedstock for ethanol production by Kluyveromyces marxianus and Saccharomyces cerevisiae. World J. Microbiol. Biotechnol. 30 (12), 3173-3183. DOI: 10.1007/s11274-014-1745-6

Malainine M.E., Dufresne A., Dupeyre D., Mahrouz M., Vuong R. y Vignon M.R. (2003). Structure and morphology of cladodes and spines of Opuntia ficus-indica. Cellulose extraction and characterisation. Carbohydr. Polym. 51 (1), 77-83.

DOI: $10.1016 / \mathrm{S} 0144-8617(02) 00157-1$

Miller G.L. (1959). Use of dinitrosalicylic acid reagent for determination of reducing sugar. Anal. Chem. 31, 426-428.
Mood S.H., Golfeshan A.H., Tabatabaei M., Jouzani G.S., Najafi G.H., Gholami M. y Ardjmand M. (2013). Lignocellulosic biomass to bioethanol, a comprehensive review with a focus on pretreatment. Renew. Sust. Energ. Rev. 27, 77-93.

DOI: 10.1016/j.rser.2013.06.033

Naik S.N., Goud V.V., Rout P.K. y Dalai A.K. (2010). Production of first and second-generation biofuels: A comprehensive review. Renew. Sust. Energ. Rev. 14, 578-597.

DOI: 10.1016/j.rser.2009.10.003

Ojumu T.V y Ogunkunle O.A. (2005). Production of glucose from lignocellulosic under extremely low acid and high temperature in batch process, auto-hydrolysis approach. J. Applied Sci. 5 (1), 15-17

Porzio G.F., Chiaramonti, D., Prussi M. y Pari L. (2012). Modelling lignocellulosic bioethanol from poplar: Estimation of the level of process integration, yield and potential for co-products. J. Clean. Prod. 34, 6675. DOI: $10.1016 /$ j.jclepro.2012.01.028

Ramos S.J.L., Martínez A. y Carreras N. (2014). Optimization of the digestion process of Scenedesmus sp. and Opuntia máxima for biogas production. Energy Convers. Manage. 88, 1263-1270.

DOI: 10.1016/j.enconman.2014.02.064

Retamal N., Durán J.M. y Fernández J. (1987). Ethanol production by fermentation of fruits and cladodes of prickly pear cactus [Opuntia ficus-indica (L.) Miller]. J. Sci. Food Agric. 40, 213-218.

Ribeiro E.M.O., Silva N.H., Lima F.J.L., Brito J.Z. y Silva M.P.C. (2010). Study of carbohydrates present in the cladodes of Opuntia ficus-indica (fodder palm), according to age and season. Ciênc. Tecnol. Aliment. 30 (4), 933-939

Rodríguez G.S., Martínez F.H.E., Órnelas N.J.L. y Garnica R.M.G. (2011). Optimización de la extracción del mucílago de nopal (Opuntia ficus-indica). Memorias. XIV Congreso Nacional de Biotecnología y Bioingeniería, Querétaro, 19 al 24 de junio.

Sáenz C. y Sepúlveda E. (2001). Cactus-pear juices. J. Prof. Assoc. Cactus 4, 3-10.

Sáenz C., Sepúlveda E. y Matsuhiro B. (2004). Opuntia spp. mucilage's: A functional component with industrial perspectives. J. Arid Environ. 57 (3), 275-290. DOI: 10.1016/S0140-1963(03)00106-X

Santos T.N., Damilano D.E., Gomes P.A., Bezerra L.F.C., Rodrigues S.R.F., Cordeiro S.D., Moraes A.C.A., Ardaillon S.D., de Morais J.M.A. y Simões C.M.R. (2016). Potential for biofuels from the biomass of prickly pear cladodes: Challenges for bioethanol and biogas production in dry areas. Biomass Bioenergy $85,215-222$.

DOI: 10.1016/j.biombioe.2015.12.005 
Sarkar N., Ghosh S.K., Bannerjee S. y Aikat K. (2012). Bioethanol production from agricultural wastes: An overview. Renew. Energy 37 (1), 19-27.

DOI: 10.1016/j.renene.2011.06.045

Serna F., Barrera L. y Montiel H. (2010). Impacto social y económico en el uso de biocombustibles. J. Technol. Manag. Innov. 6 (1), 100-114.

Serra A.T., Poejo J., Matias A.A., Bronze M.R. y Duarte C.M.M. (2013). Evaluation of Opuntia spp. derived products as antiproliferative agents in human colon cancer cell line (HT29). Food Res. Int. 54 (1), 892-901. DOI: 10.1016/j.foodres.2013.08.043

Sheikh M.I., Kim C.H., Lee J.Y., Kim S.H., Kim G.C., Lee J.Y., Shim S.W. y Kim J.W. (2013). Production of bioethanol from waste money bills. A new cellulosic material for biofuels. Food Bioprod. Process. 91 (1), 60-65. DOI: 10.1016/j.fbp.2012.09.001

Sims R.E.H., Mabee W., Saddler J.N. y Taylor M. (2010). An overview of second generation biofuel technologies. Bioresour. Technol. 101 (6), 1570-1580.

DOI: $10.1016 /$ j.biortech.2009.11.046

Soccol C.R., Vandenberghe L.P., Medeiros A.B., Karp S.G., Buckeridge M., Ramos L.P., Pitarelo A.P., Ferreira L.V., Gottschalk L.M., Ferrara M.A., da Silva B.E.P., de Moraes L.M., Araujo J. de A. y Torres F.A. (2010). Bioethanol from lignocelluloses: Status and perspectives in Brazil. Bioresour. Technol. 101 (13), 4820-4825.

DOI: 10.1016/j.biortech.2009.11.067

Stintzing F.C. y Carle R. (2005). Cactus stems (Opuntia spp.): A review on their chemistry, technology, and uses. Mol. Nutr. Food. Res. 49, 175-194.

DOI: $10.1002 / \mathrm{mnfr} .200400071$

Tacias P.V.G., Rosales Q.A. y Torrestiana S.B. (2016). Evaluación y caracterización de grasas y aceites residuals de cocina para la producción de biodiésel: un caso de estudio. Rev. Int. Contam. Ambie. 32 (3), 303-313. DOI: 10.20937/RICA.2016.32.03.05

Taherzadeh M.J. y Karimi K. (2007). Enzyme-based hydrolysis processes for ethanol from lignocellulosic materials: A review. Bioresources 2 (4), 707-738.
Taherzadeh M J. y Karimi K. (2008). Pretreatment of lignocellulosic wastes to improve ethanol and biogas production: A review. Int. J. Mol. Sci. 9 (9), 1621-1651. DOI: $10.3390 /$ ijms 9091621

TAPPI (2009). Norma T222 om-88. Acid insoluble lignin in wood and pulp. Technical Association of the Pulp and Paper Industry, Atlanta, GA, EUA.

Tayeh H.A., Najami N., Dosoretz C., Tafesh A. y Azaizeh H. (2014). Potential of bioethanol production from olive mill solid wastes. Bioresour. Technol. 152, 24-30. DOI: 10.1016/j.biortech.2013.10.102

Vallejos M.E., Zambon M.D., Area M.C. y da Silva C.A.A. (2015). Low liquid-solid ratio fractionation of sugarcane bagasse by hot water autohydrolysis and organosolv delignification. Ind. Crops Prod. 65, 349353. DOI: 10.1016/j.indcrop.2014.11.018

Vargas B.G.J. y Pereira N. (2010). Sugar cane bagasse as feedstock for second generation ethanol production. Part I: Diluted acid pretreatment optimization. Electron. J. Biotechnol. 13 (5), 2-9.

DOI: $10.2225 /$ vol13-issue 5-fulltext-8

Xiao X., Bian J., Peng X.P., Xu H., Xiao B. y Sun R. C. (2013). Autohydrolysis of bamboo (Dendrocalamus giganteus Munro) culm for the production of xylooligosaccharides. Bioresour. Technol. 138, 63-70.

DOI: 10.1016/j.biortech.2013.03.160

Ximenes E., Kim Y., Mosier N., Dien B. y Ladisch M. (2010). Inhibition of cellulases by phenols. Enzyme Microb. Technol. 46 (3-4), 170-17. DOI: $10.1016 / j$.enzmictec.2009.11.001

Yang L., Lu M.I.., Carl S., Mayer J.A., Cushman J.C., Tian E. y Lin H. (2015). Biomass characterization of Agave and Opuntia as potential biofuel feedstocks. Biomass Bioenergy 76, 43-53.

DOI: 10.1016/j.biombioe.2015.03.004

Zhu M.Q., Wen J.L., Su Y.Q., Wei Q. y Sun R.C. (2015). Effect of structural changes of lignin during the autohydrolysis and organosolv pretreatment on Eucommia ulmoides Oliver for an effective enzymatic hydrolysis. Bioresour. Technol. 185, 378-385.

DOI: 10.1016/j.biortech.2015.02.061 